\title{
Histopathological and ultrastructural examination of optic nerve sheath decompression
}

\author{
James C Tsai, Margaret S Petrovich, Alfredo A Sadun
}

Doheny Eye Institute and the Department of Ophthalmology, University of Southern California School of Medicine, Los Angeles, USA

J C Tsai

M S Petrovich

A A Sadun

Correspondence to: Dr Alfredo Sadun, Doheny Eye Institute, 1450 San Pablo Street, USA.

Accepted for publication 5 September 1994 Los Angeles, CA 90033,

\begin{abstract}
Both optic nerves were obtained at autopsy from a 28-year-old man with a 2 year history of idiopathic intracranial hypertension who had undergone bilateral optic nerve sheath decompression 14 days before death. Histopathological and ultrastructural examination of the tissue revealed fibroblasts localised to the sites of fenestration. Adipose tissue was also adherent to the optic nerve pia in areas of incised dura. No patent fistula site was observed. It was concluded that filtration of cerebrospinal fluid after optic nerve sheath decompression may occur through an enclosed bleb of fibrosis rather than through an open fistula.

(Br f Ophthalmol 1995; 79: 182-185)
\end{abstract}
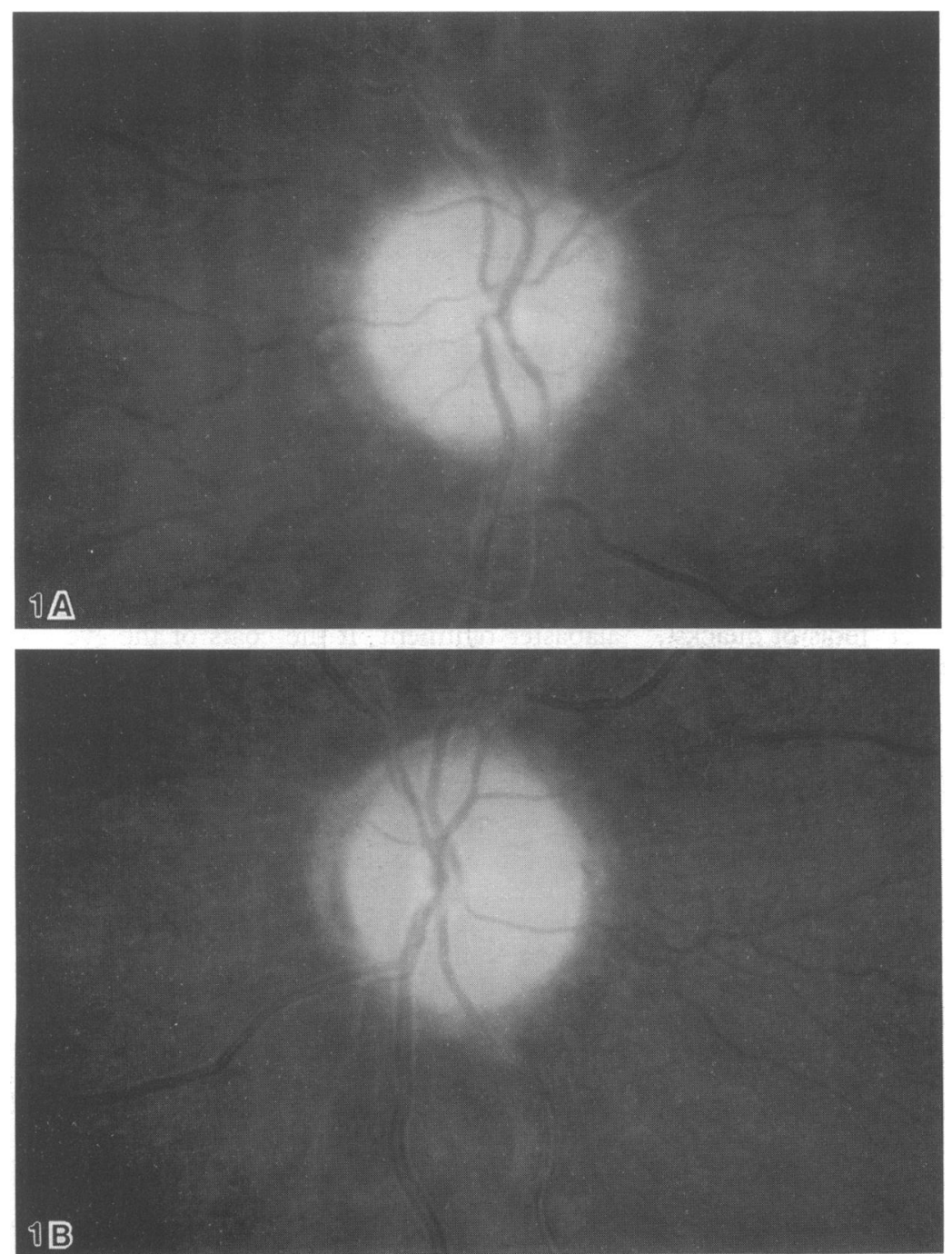

Figure 1 Optic disc photographs of the right eye $(A)$ and left eye $(B)$ immediately before surgery. Note presence of secondary optic atrophy in both discs.
Optic nerve sheath decompression (ONSD) has been reported to be effective in preserving vision in patients with idiopathic intracranial hypertension (that is, pseudotumour cerebri) ${ }^{1-3}$ and progressive anterior ischaemic neuropathy. 4 At present, its exact mechanism of action has not been clearly defined, and few histopathological studies of optic nerve specimens after sheath decompression have been described. ${ }^{5-9}$ Keltner and colleagues ${ }^{5} 6$ reported the presence of intact fistulas at the sites of fenestration, and concluded that the mechanism of action is the successful egress of cerebrospinal fluid. Davidson ${ }^{78}$ observed secondary fibrosis surrounding the incision sites, and suggested that this scarring phenomenon ultimately protects the optic disc from the more proximal elevated intracranial pressure.

We report here the histopathological and ultrastructural finding in both optic nerves from a patient with chronic idiopathic intracranial hypertension who died 14 days after undergoing bilateral ONSD.

\section{Case report}

A 28-year-old right handed man was initially referred to our institution with a 3 week history of transient obscurations of vision, bifrontal and bitemporal headaches, and dimming of vision. The patient had otherwise been in excellent health except for morbid obesity of at least 10 years' duration. Visual acuity was $20 / 200$ in the right eye and 5/200 in the left eye. Colour vision was markedly diminished in the left eye, but he could discern $7 \cdot 5$ of 8 pseudoisochromatic plates (American Optical) with the right eye. Brightness sense in the left eye was $28 \%$ of that in the right eye. There was a marked afferent pupillary defect of the left eye. Tangent field testing, performed with a $10 \mathrm{~mm}$ white target, showed marked constriction of the peripheral field in the right eye. The left eye showed a central altitudinal field defect in addition to peripheral field constriction. Dilated fundus examination revealed florid bilateral disc oedema, worse in the left eye, and marked oedema of the peripapillary retina.

The patient was diagnosed as having papilloedema with severe impairment of vision most probably secondary to idiopathic intracranial hypertension. A computed tomography scan of the brain with contrast was normal. A lumbar puncture revealed an opening pressure of $420 \mathrm{~mm} \mathrm{Hg}$. Results of laboratory studies including cerebrospinal fluid studies were all within normal limits except for an elevated erythrocyte sedimentation rate of $65 \mathrm{~mm} / \mathrm{h}$ (Westergren). 

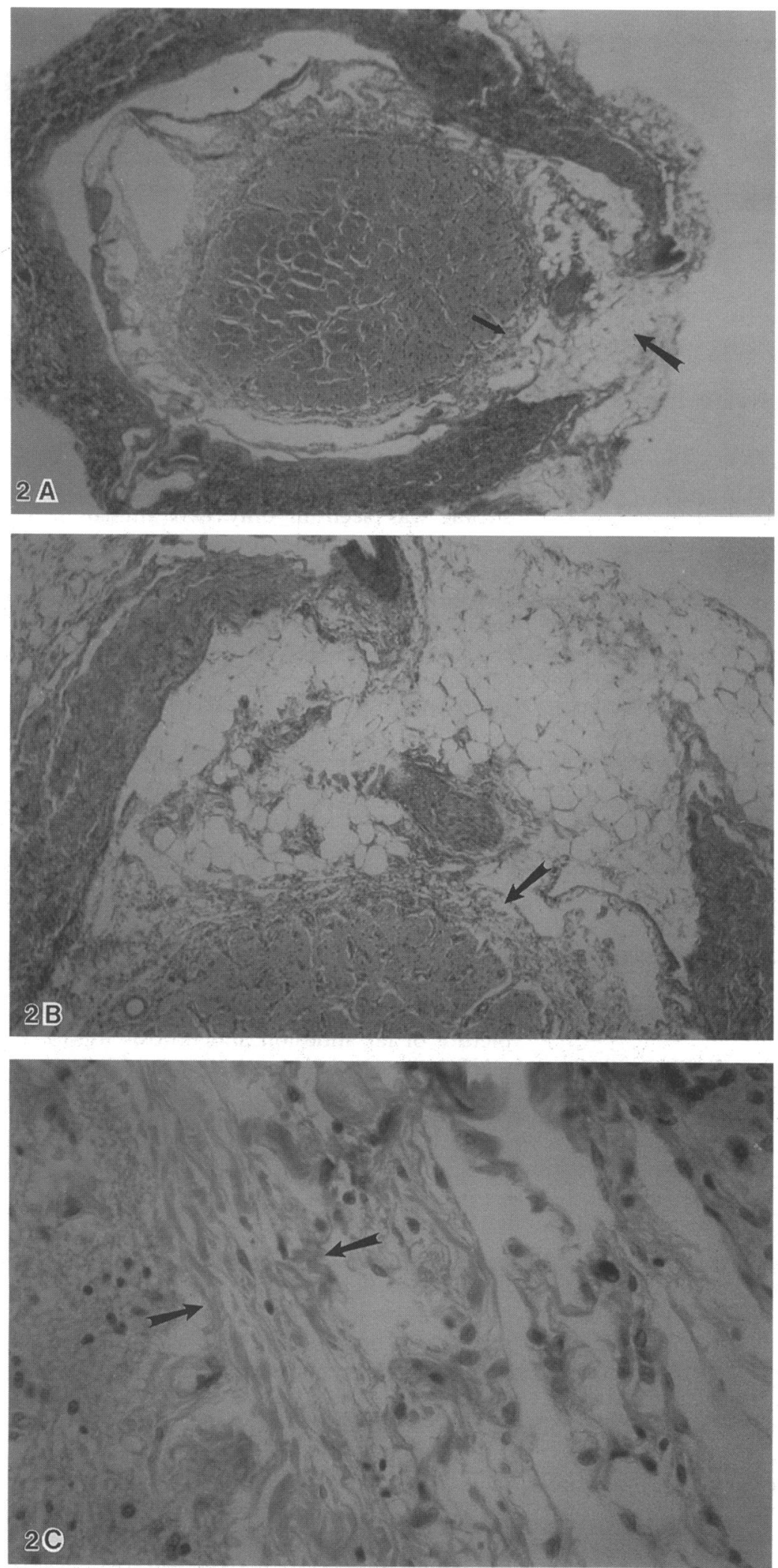

Figure 2 Light micrographs of one of the fenestration sites (trichrome stain). (A) Low magnification view $(\times 2)$ of the optic nerve in cross section. Note that the fenestration site traverses the dura and arachnoid into the subarachnoid space. The pia (small arrow) is intact. Adipose tissue (large arrow) is adherent to pia in areas of incised dura. (B) High magnification view ( $\times 4.75)$ demonstrates proliferation of granulation tissue and the adherence of adipose tissue to the pia (arrow) in areas of fenestration. A peripheral ciliary nerve is located centrally. No discernible filtration bleb is seen. (C) Highest magnification view $(\times 75)$ reveals no obvious fistula site between the pia and the overlying fibrosis (see arrows).
(15 degrees of central vision in the right eye and 10 degrees in the left eye by static visual field testing). Fundus examination revealed secondary optic atrophy with characteristic gliosis of the optic discs.

Two years later the patient was referred again to our neuro-ophthalmology clinic. He had $20 / 25$ vision in both eyes with constricted visual fields, moderate disc oedema with secondary optic atrophy (Fig 1), and marked Cushingoid features. A relative afferent pupillary defect was not present. The patient identified 6 of 8 pseudoisochromatic plates with his right eye and 5 of 8 plates with his left eye. In view of progressive visual field loss, the continued increased intracranial pressures, and the dangers of prolonged corticosteroid use, we recommended bilateral ONSD in order to begin immediately tapering off the corticosteroids.

The patient underwent bilateral ONSD by a medial approach. Five separate longitudinal incisions were made into the nerve sheaths bilaterally with either a ruby knife or supersharp blade. Each incision was made from a point 2-3 $\mathrm{mm}$ posterior to the globe and extended $5 \mathrm{~mm}$ distally along the nerve sheath. Clear cerebrospinal fluid (upon which floated small yellow oil drops) emanated from the exposed subarachnoid space.

Four days postoperatively, the patient immediately reported seeing more clearly and had resolution of his headaches but now noted diplopia. Visual acuity was $20 / 25$ in the right eye and $20 / 30$ in the left eye. He identified 7 of 8 pseudoischromatic plates with each eye, and his colour vision was subjectively much better. The pupils were briskly reactive to light and near target, without evidence of an afferent defect. Funduscopic examination revealed mild disc oedema. The patient was begun on an acute taper of his oral corticosteroids.

Approximately 2 weeks later, he had gastrointestinal haemorrhaging that proved to be fatal. An autopsy was performed, during which both eyes and optic nerves were removed for microscopic and ultrastructural study.

\section{Results}

Each eye measured $24.5 \times 23.5 \times 24.0 \mathrm{~mm}$, appeared normal externally, and had approximately $15 \mathrm{~mm}$ of optic nerve attached. Gross examination of the sectioned globes revealed diffuse secondary atrophy of the optic nerves bilaterally. There was no evidence of disc oedema. No discernible filtration blebs were noted along the fenestration sites. The optic nerves were adjacently sectioned and then stained with haematoxylin and eosin, luxol fast blue, trichrome, and Bodian stains.

Light microscopy with multiple coronal and sagittal sections revealed severe optic atrophy with only a small percentage of axons remaining near the centre of each optic nerve. Localised proliferation of connective tissue was seen overlying the fenestration sites throughout the entire nerve and were present in all levels of the sections (Fig 2). Adipose tissue
The initial treatment regimen included acetazolamide and steroids, weekly lumbar punctures, and an intensive diet programme. Visual acuity returned to $20 / 20$ in both eyes but with residual peripheral field constriction 


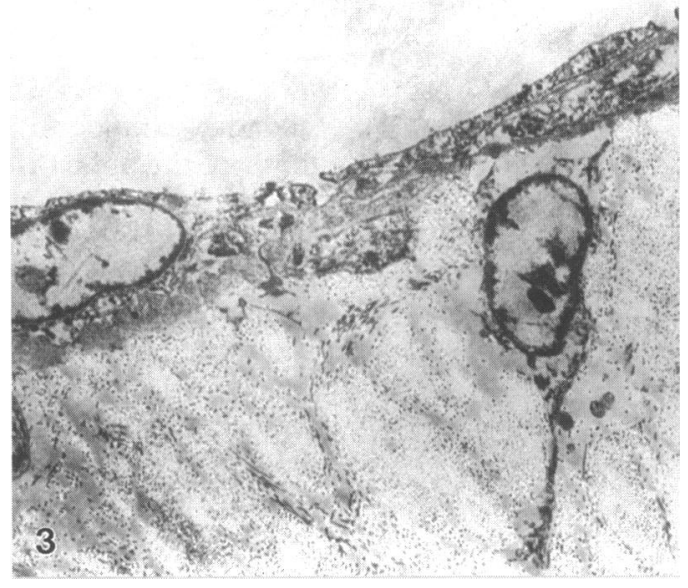

Figure 3 Transmission electron micrograph of the edge of the peripheral ciliary nerve depicted in Figure 2. Fibroblast cells are seen; one of them is seen entering the surrounding collagen matrix (magnification $\times 3900)$.

was noted to be adherent to the optic nerve pia in areas of incised dura. No patent fistula site was observed in any of the specimens of either optic nerve. Regions of the nerve other than the fenestration sites did not reveal any fibrovascular proliferation or adherent fat. There was no histological evidence of inflammation in the orbital fat, muscles, or blood vessels.

Ultrastructural examination clearly demonstrated the extent of this fibrotic response (Fig 3). Endothelial cells lined the edge of the peripheral ciliary nerve. Many fibroblasts were seen within the surrounding collagen matrix.

\section{Discussion}

Even though ONSD has been performed since the late nineteenth century for relief of papilloedema, ${ }^{10}$ its exact mechanism of action remains obscure. Davidson ${ }^{78}$ proposed that the relief of papilloedema after ONSD was attributable to an obliteration of the subarachnoid space by fibroblasts, which prevented transmission of the increased intracranial pressure along the subarachnoid space. The patient described by Davidson ${ }^{7}$ in 1969 survived for 21 days after surgery, and histopathological examination of postmortem tissues revealed occlusion of the dural incision sites with 'young granulation tissue' and the presence of 'organised granulation tissue with some fat necrosis.' Another patient reported in $1972^{8}$ underwent ONSD approximately 12 days before death. Histological examination showed that the incised dura was plugged by fibroblasts that arose from the subarachnoid space. It is important to note that Davidson believed that occlusive fibrosis was responsible for the initial reduction in disc oedema in this patient, though the optic nerve specimen was taken 1 week after this patient's vision had decreased from preoperative levels.

In 1977 Keltner et al ${ }^{6}$ reported a histological study of the optic nerves of a patient who died 39 days after nerve sheath decompression for intractable chronic papilloedema. The presence of intact fistulas in the dura and an open subarachnoid space around the optic nerve led them to propose that the mechanism of action of ONSD was primarily that it allowed egress of cerebrospinal fluid (CSF). They concluded that the flow of CSF through the loose arachnoid-like connective tissue that proliferated into the fistulas accounted for resolution of the papilloedema. Moreover, this increased resistance, open shunt mechanism could account for the clinically observed rapidity of onset of symptomatic improvement and bilateral diminution of disc oedema following unilateral ONSD.

Hayreh $^{9}$ performed a histological study of the optic nerve in nine monkeys with induced papilloedema that had undergone ONSD. Examination revealed proliferation of connective tissue with adherence to the pia at the sites of decompression. Closure of the incised nerve sheath was seen in only two animals, and resulted from invasion of the deeper layers of optic nerve by the proliferative connective tissue. No temporal relation could be found for this exaggerated fibrosis response since open sheath sites were seen in some animals at 31 to 63 days after surgery, while in the other two animals closed sheath sites were noted after 27 and 40 days, respectively.

In our patient there were localised regions of proliferative fibrosis overlying the fenestration sites throughout the nerve, and adipose tissue was noted to be adherent to the optic nerve pia in areas of incised dura, consistent with the observations of Davidson ${ }^{8}$ and Hayreh. ${ }^{9}$ No patent fistula was identified. In areas other than the sites of fenestration, we did not note either adherent fat or fibrovascular proliferation. We conclude that the histopathological picture of fat adhesion and fibrosis following ONSD is consistent with a mechanism of closed filtration of CSF (that is, analogous to aqueous filtration through an enclosed trabeculectomy bleb) rather than filtration through an open fistula.

We believe, as did Keltner and colleagues, ${ }^{56}$ that egress of CSF is the initial mode of action of ONSD. However, we hypothesise that this filtration of fluid occurs through an enclosed bleb of proliferative fibrosis, rather than through an open fistula. The fibrotic response seen in our optic nerve specimens may be similar to the proliferation of connective tissue at the fenestration sites observed by Hayreh. ${ }^{9}$ The high dose corticosteroids received by our patient in the immediate postoperative period may also have diminished the extent of the proliferative fibrosis. Closure of the incised dura sites noted by Hayreh ${ }^{9}$ in two of his monkeys, and seen also by Davidson ${ }^{78}$ in two human patients may represent excessive fibrosis around the incision site, such as seen when a bleb becomes flattened and fibrosed after glaucoma filtering surgery. In fact, Davidson's histopathological description of fibrotic scarring at the incision sites in one patient ${ }^{8}$ may have represented failure of a previously functioning filtering bleb that had been responsible for the initial reduction in disc oedema noted in the first 5 postoperative days.

In conclusion, our histopathological and ultrastructural study may further elucidate the 
mechanism of action of ONSD. We believe that the majority of successful cases of ONSD result from the continual egress of CSF through an enclosed bleb, rather than through an obvious fistula. Excessive fibrosis may eventually undermine the function of this filtration bleb and lead to total closure of the incised dura and surrounding subarachnoid space.

This study was supported in part by Core grant EY03040 from the National Eye Institute, Bethesda, MD, USA

Dr Tsai is currently affiliated with Moorfields Eye Hospital, London, England.

Presented in part at the Annual Meeting of the Association for Research in Vision and Ophthalmology, Sarasota, FL, 7 May, 1993.

We thank Ann Dawson, MLA, for editorial assistance and Narsing A Rao, MD, for his assistance in interpreting the light and transmission electron micrographs.

1 Brourman ND, Spoor TC, Ramocki JM. Optic nerve sheath decompression for pseudotumor cerebri. Arch Ophthalmol 1988; 106: 1378-83.
2 Sergott RC, Savino PJ, Bosley TM. Modified optic nerve sheath decompression provides long-term visual improvement for pseudotumor cerebri. Arch Ophthalmol 1988, 106: 1384-90.

3 Corbett JJ, Nerad JA, Tse DT, Anderson RL. Results of optic nerve sheath fenestration for pseudotumor cerebri: the lateral orbitotomy approach. Arch Ophthalmol 1988; 106: 1391-7.

4 Sergott RC, Cohen MS, Bosley TM, Savino PJ. Optic nerve decompression may improve the progressive form of nocompression may improve the progressive form of 1989; 107: 1743-54.

5 Keltner JL. Optic nerve sheath decompression. How does it work? Has its time come? Arch Ophthalmol 1988; 106: 1365-9.

6 Keltner JL, Albert DM, Lubow M, Fritsch E, Davey LM. Optic nerve decompression: a clinical pathologic study. Arch Ophthalmol 1977; 95: 97-104.

7 Davidson SI. A surgical approach to plerocephalic disc oedema. Trans Ophthalmol Soc UK 1969; 89: 669-90.

8 Davidson SI. The surgical relief of papilloedema. In: Cant JS, ed. The optic nerve. London: Henry Kimpton, 1972; 174-9. The optic nerve. London: Henry Kimpton, 1972;

9 Hayreh SS. Pathogenesis of oedema of the optic disc. Doc Ophthalmol 1968; 24: 289-411.

10 Galbraith JEK, Sullivan JH. Decompression of the perioptic meninges for relief of papilledema. Am $\mathcal{f}$ Ophthalmol 1973; 76: 687-92. 